\title{
A new journal to address an old problem: experimental stroke, lack of neuroprotective agents and translational medicine
}

\author{
Bruno P. Meloni, PhD*, Kym Campbell, BVMS, Neville W. Knuckey, MD \\ Centre for Neuromuscular and Neurological Disorders, University of Western Australia, \\ Australian Neuromuscular Research Institute, \\ Department of Neurosurgery, Sir Charles Gairdner Hospital, \\ Nedlands, Western Australia, Australia.
}

It is with great pleasure and enthusiasm that we take the opportunity to introduce the new free-access online peer-reviewed periodical Journal of Experimental Stroke and Translational Medicine (JESTM). The creation of this journal is largely the brainchild of Dr Shimin Liu, and he should be highly commended for all his efforts in bringing it to fruition. As its title suggests, the journal is intended principally to gather and disseminate new knowledge in the field of experimental stroke in order to facilitate future translation and development of new clinical stroke treatments. The creation of the journal is timely as in recent years there has been increasing concern over the failure of neuroprotective therapeutic modalities that have been shown to be effective in animal studies but that have failed at clinical trial. It is anticipated that the journal will help address the issues that have hampered this exciting and challenging field by publishing experimental, review and opinion articles: i) promoting the understanding of stroke injury mechanisms, ii) identifying and characterising new therapeutic targets, iii) providing recommendations for the preclinical assessment of neuroprotective agents, and iv) reporting the results of the preclinical assessment of new neuroprotective therapies.

Fittingly, in the first issue (Volume 1) Dr Liu, in a review article entitled "The Continued Promise of Neuroprotection for Acute Stroke Treatment"

\footnotetext{
* Corresponding Author and to whom correspondence and proofs should be sent to:

Bruno P. Meloni

Centre for Neuromuscular and Neurological Disorders

University of Western Australia

4 Floor, A Block

QEII Medical Centre

Verdun Street, Nedlands WA 6009

Tel: 61893463535

Fax:618 93463487

Email: meloni@cyllene.uwa.edu.au

Copyright $\odot 2009$ SFES 1939-067X/09
}

discussed some fundamental issues concerning the translational failure of experimental neuroprotective treatments to the clinical setting. In doing so, he highlighted the importance of restoring and preserving the energy status of ischaemic cells/tissue as a primary objective to promoting survival. If this can be achieved effectively, and especially if combined with other strategies targeting downstream events (e.g., activation of pro cell death genes/proteins), the likelihood of discovering successful neuroprotection after stroke will no doubt be greatly enhanced.

The current issue (Volume 2) comprises one experimental study, one epidemiological study and three review articles. The experimental study by Cross et al concerns the role of the sodium calcium exchange protein $\mathrm{NCX} 3$ in neuronal injury as assessed in in vitro and animal stroke models. The role of NCX isoforms is of considerable interest as the exchanger can transport calcium either into or out of cells, and hence its role in brain injury following stroke might exacerbate or ameliorate calcium dysregulation. Cross et al provide in vitro and in vivo data that suggest that NCX activity is beneficial during mild ischemia by maintaining calcium homeostasis, but that during severe ischaemia it may be contributing to calcium overload and cell death. In their epidemiological study, Huang et al present their findings on identifying early and distinctive cognitive predictors of vascular dementia in an elderly Chinese population. With an aging population the importance of identifying earlier predictors of dementia is vital in order to provide the opportunity for early intervention with existing and future therapies.

The review articles cover diverse topics; publication bias, delivery of peptides to the brain and the role of NADPH oxidase in ischemic brain injury. $\mathrm{Dr}$ Liu discusses the problem of publication bias in the scientific literature, how it can be identified, why it arises and how the issue can be addressed. The 
need to minimise bias in experimental stroke studies is emphasised, as is one of the journal's goals, namely its willingness to review manuscripts containing "controversial/provocative ideas and negative results". The Meade et al review highlights the potential advantages of proteins and peptides as neuroprotective therapeutics, considers their delivery to the brain by means of cell penetrating peptides (CPPs) and gathers together studies that have used this approach in experimental stroke models. As the blood brain barrier (BBB) is a major obstacle in terms of drug access to the brain, the use of CPPs to deliver peptides/proteins and other therapeutic cargo into this well-guarded organ has great clinical potential in stroke and other central nervous systems disorders. Finally, Dr Chen provides a concise article outlining the structure of the pro-oxidant multi-subunit $\mathrm{NADPH}$ oxidase protein, its role in astrocyte and microglia function, long-term potentiation and in learning, as well as its effects in the brain following ischemia. With respect to the latter, $\mathrm{Dr}$ Chen draws upon data from both in vitro and in vivo studies to dissect out the damaging roles played by NADPH oxidase at the cellular/tissue (neurons, microglia) and BBB (astrocytes/endothelium, matrix metalloproteinases) levels.

In only its first two issues, the journal is already showing great promise as a dedicated forum to generate an open and ongoing discussion that can only improve the quality of research in this increasingly important field. We believe the journal will be of interest to all investigators involved in any aspect of stroke research, whether preclinical or clinical. We congratulate all of the contributors, particularly Dr Liu, for providing a solid foundation on which to build this ambitious journal's success, and we encourage the stroke research community to make full use of this very welcome resource. 